\title{
Effectiveness of Brain Protection With Histidine-Tryptophan-Ketoglutarate Solutions
}

\author{
Sonay Oğuz, ${ }^{1}$ Halil F. Aşgün, ${ }^{1}$ Başak Büyük ${ }^{2}$ \\ ${ }^{1}$ Department of Cardiovascular Surgery, Faculty of Medicine, Canakkale Onsekiz Mart University, Canakkale, Turkey; ${ }^{2}$ Department \\ of Histology and Embryology, Faculty of Medicine, İzmir Democracy University, İzmir, Turkey
}

\section{ABSTRACT}

Background: Tissue-protective solutions increase resistance of cells to ischemic conditions. Especially in carotid and aortic arch surgeries where the brain perfusion is at risk, these solutions may be beneficial to prevent ischemic brain damage. This study was designed to demonstrate the effectiveness of histidine-tryptophanketoglutarate (HTK) solution in increasing resistance of brain tissue to ischemic conditions.

Methods: Three separate randomized groups were created, each consisting of eight rabbits. The groups were called the ischemia, HTK and sham groups, respectively. In the ischemia group, temporary brain ischemia was created for 15 minutes by placing clamps on the bilateral subclavian and common carotid arteries. Then the clamps were removed, and the brain was reperfused for 30 minutes. In the HTK group, HTK solution was sent to the brain through the internal carotid artery before the same ischemia-reperfusion protocol was applied. Histopathological analyses using a visual scoring system to assess the degree of ischemic changes and the apoptotic cell index by TUNEL test were performed in all brain tissue samples.

Results: Apoptotic cell indices of the HTK (20.6\%) and sham $(17.8 \%)$ groups were lower than the ischemia group $(56.8 \%)(P<.05)$. Statistically significant differences were detected between all groups in categorical scores $(P<.05)$.

Conclusions: It was shown that less ischemic damage occurs in the brain tissue with the use of HTK solution, and it may be a candidate approach to prevent the brain from ischemic insults during cerebrovascular surgery. Further studies are required to demonstrate its exact effectiveness, in terms of dose, duration, and temperature.

\section{INTRODUCTION}

Although lack of suitable perfusion causes cell death and tissue damage as seen in stroke or heart attack, tissue may become more resistant to ischemia in some situations or with

Received May 30, 2020; accepted Fune 18, 2020.

Correspondence: Sonay Oğuz, Department of Cardiovascular Surgery, Faculty of Medicine, Canakkale Onsekiz Mart University, Barbaros Street Terzioglu Campus B Block No. 4, 17100, Canakkale, Turkey (e-mail: soguz@comu.edu.tr). some treatments. An example of this protective strategy is the use of organ-protective solutions in transplantation surgeries. This effect is provided by amino acids, which are known to increase cell resistance against ischemia and reperfusion injury [Kalogeris 2012; Saitoh 2000]. Efficacy of these amino acids, especially the so-called HTK solutions containing Histidine, Tryptophan, and Ketoglutarate, has been proven [Arslan 2005; Lee 2011; Lee 2010; Saitoh 2000; Michel 2002]. In addition, their routine use has been reported in heart, kidney, liver and pancreas transplantation, due to its beneficial effects against ischemic tissue damage [Agarwal 2006; Edelman 2013; Englesbe 2006; Prathanee 2015; Ringe 2005; Voigt 2013].

The development of neurologic sequelae as a consequence of cerebral ischemia still continues to be a serious health problem. There is not enough information in the literature regarding the use of HTK solutions to increase the resistance of brain tissue to ischemia. Cerebral circulation is reduced and even ceased in some type of surgeries, including carotid artery surgery, deep hypothermic circulatory arrest, and arcus aorta surgery. Although various brain protection strategies have been suggested for these surgeries, neurologic damage secondary to intervention continues to be a major problem [Gokalp 2019; Goodney 2012; Iscan 2018; Okita 2015]. In these cases, brain protection with infusion of these solutions through the carotid artery may become an option. These surgeries are suitable to send HTK solution directly to the brain tissue through the carotid artery for an improved resistance to cerebral ischemia. However, there are not enough studies on this subject in the literature. For this purpose, this animal experiment was designed. In this study, the effects of HTK solution, which was sent directly into the carotid artery of rabbits to protect the brain tissue against ischemia was evaluated by histopathological examination.
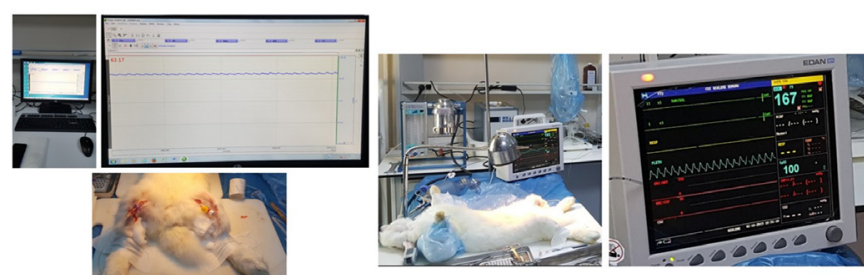

Figure 1. The monitorization of rabbits. 


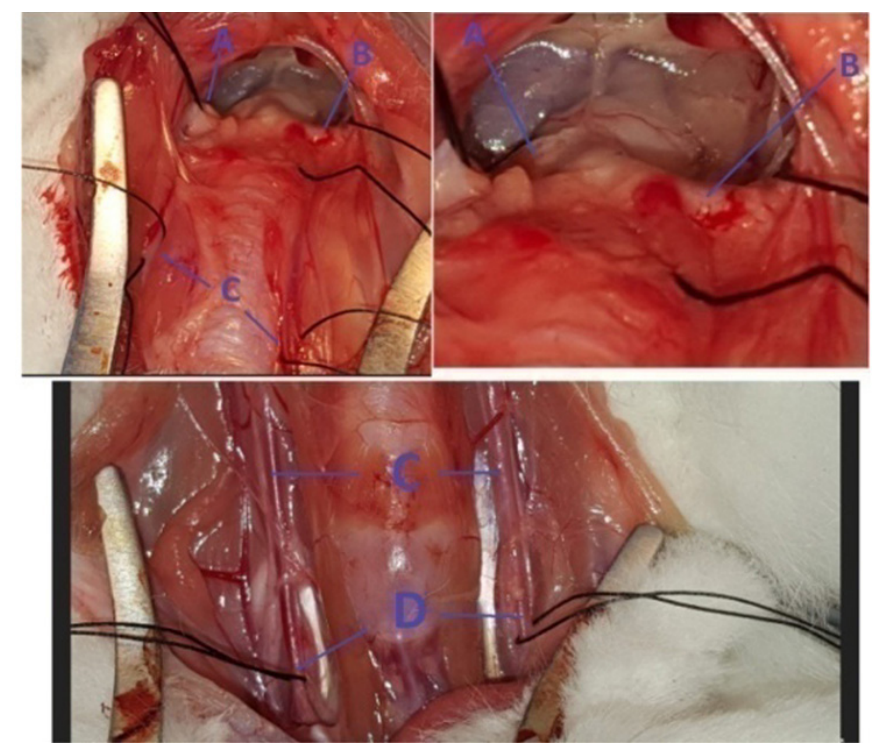

Figure 2. A, left subclavian artery, B, right subclavian artery, C, bilateral common carotid arteries, D, bilateral external carotid arteries.

\section{METHODS}

Experimental animals: Twenty-four New Zealand male rabbits aged between 11 months and 13 months and weighing between 2.3 and $3.4 \mathrm{~kg}$ were used in the study. Standard rabbit feed and water were provided throughout the study. The rabbits were individually housed in a special steel cage in a room at $22 \pm 2^{\circ} \mathrm{C}$ and $55-60 \%$ humidity with a 12-h dark/ light cycle.

Study groups: The rabbits were randomized into the following three groups: ischemia, HTK, and sham. With the ischemia group $(\mathrm{N}=8)$, temporary artificial brain ischemia was created for 15 minutes by placing clamps on the bilateral subclavian and common carotid arteries. Then the clamps were removed and brain reperfused for 30 minutes. With the HTK group $(\mathrm{N}=8)$, the external carotid arteries, bilateral subclavian and common carotid arteries were clamped after bilateral main carotid artery cannulation. HTK solution was sent to the brain tissue through the internal carotid artery via cannulas. After the end of HTK infusion, clamping was continued and experimental brain ischemia obtained for another 15 minutes. The clamps were removed and a 30-minute reperfusion period was completed. The sham group $(\mathrm{N}=8)$ had the same surgical preparation as the HTK group. The preparation was performed in the rabbits without vascular occlusion and intraarterial infusion. After the procedures were completed in all groups, the whole brain was rapidly removed from each rabbit and sent to histopathological study.

Anesthesia protocol and monitoring: After the premedication of xylazine $(10 \mathrm{mg} / \mathrm{kg})$, anesthesia induction was achieved by intramuscularly applying ketamine hydrochloride $(40 \mathrm{mg} /$ $\mathrm{kg})$. Maintainance of anesthesia was obtained by isoflurane inhalation with a mixture of $\mathrm{FiO} 260 \%$. Vital signs of the rabbits, including oxygen with a pulse oximeter ( $\operatorname{Im} 8$, Edan, San

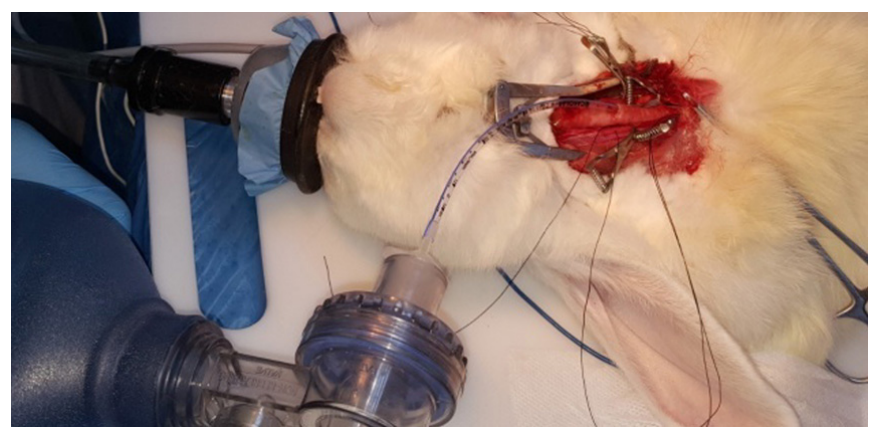

Figure 3. The endotracheal tube and vascular occlusion by the clamps.

Diego, California, USA) placed in the ear and invasive arterial blood pressure (Mp36r, Biopac, Goleta, California, USA) via a catheter placed in femoral artery, were followed. Vascular access was provided with a catheter placed in the opposite femoral vein (Figure 1).

HTK solution properties, preparation, and application: There are solutions containing histidine, tryptophan, ketoglutarate, which have known tissue protection properties. PlegiStore ${ }^{\circledR}$ brand HTK solution was used in the experiment. It contains $180 \mathrm{~mm} / \mathrm{L}$ histidine, $2.0 \mathrm{mmol} / \mathrm{L}$ tryptophan, 1.0 $\mathrm{mmol} / \mathrm{L}$ potassium hydrogen 2-ketoglutarate, $30.0 \mathrm{mmol} / \mathrm{L}$ mannitol, $15.0 \mathrm{mmol} / \mathrm{L}$ sodium chloride, $9.0 \mathrm{mmol} / \mathrm{L}$ potassium chloride, and $4.0 \mathrm{mmol} / \mathrm{L}$ magnesium chloride per 1000 $\mathrm{mL}$ bag. The product was kept at $4-10^{\circ} \mathrm{C}$ during transportation, storage, and application. It was administered at a perfusion rate of $1 \mathrm{~mL} / \mathrm{gr} / \mathrm{min}$ from a $50-70 \mathrm{~cm}$ height to obtain a perfusion pressure equivalent to $40-50 \mathrm{mmHg}$ within a 8 - to 10-minute infusion period through the catheters placed into both common carotid arteries. The solution was applied by leaving it at free flow from a fixed height to avoid intracranial pressure elevation and hemorrhage. Brain tissue weight was detected in the range of 8-10 grams in the sham and ischemia groups. The amount of solution was adjusted in the HTK group, according to this brain weight.

Surgical procedures: After anesthesia was provided, the neck, ear and legs of the rabbits were shaved. The arms and legs were fixed with the help of adhesive tape patches to provide a flat supine position. A median skin incision was performed from the upper part of the sternum to the anterior cervical region up to the epiglottic level. Bilateral common carotid arteries and bilateral subclavian arteries were found and prepared for clamping (Figure 2). In addition, the external carotid arteries were prepared for clamping in the HTK group. Local prilocaine $0.5 \mathrm{~mL}$ was applied to the carotid bulbous region to provide baroreceptor blockade. To prevent thrombus formation in all subjects, unfractionated heparin intravenously was administered 5 minutes before arterial clamping (100 unit $/ \mathrm{kg})$.

Ischemia group: The brain circulation was interrupted by clamping the bilateral subclavian and bilateral main carotid arteries with a mini vascular clamp for 15 minutes (Figure 3).

After 15 minutes of brain ischemia, clamps were removed and reperfusion was achieved for 30 minutes. Hemodynamics closely were monitored to prevent hypotension. After the 


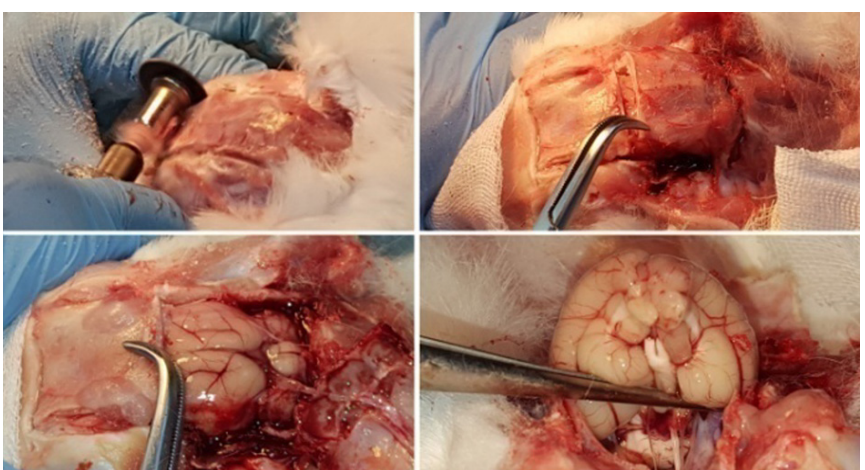

Figure 4. The complete removal of the brain.

30-minute reperfusion period, total brain tissue was removed and prepared for histological examination (Figure 4).

HTK group: Following the arterial preperation in the ischemia group, thin catheters $(26 \mathrm{G})$ were placed into the bilateral common carotid artery (Figure 5A), and the external carotid arteries were prepared for clamping at the bifurcation level (Figure 2D). Mini clamps were placed on the bilateral subclavian arteries, bilateral common carotid arteries, and bilateral external carotid arteries (Figure 5B). Subsequently, prepared HTK solution was infused within 8-10 minutes through the catheters placed into the common carotid arteries. (Figure 5C) Mean arterial pressure was observed to maintain above $50 \mathrm{mmHg}$ by invasive artery monitoring. After cerebral infusion of HTK solution was over, the 15-minute brain ischemia period was continued without removing the clamps. (Figure 5D) Afterward, clamps were removed, and the 30-minute reperfusion period was completed. At the end of the procedure, each rabbit's brain was quickly removed and prepared for histological examination. An endotracheal tube (Cuffless ET-3.0 cutters, Bicakcilar, Turkey) was inserted below the larynx before respiratory depression occurred, due to brain ischemia in the ischemia and HTK groups, and manual ventilation was provided.

Sham group: The surgical preparation described above was done. However, the arteries were not clamped, and the brains were removed in the same manner without additional process.

Histopathological examination: After the surgical procedure was complete, the brains were fixed in $10 \%$ neutral buffered formaldehyde. Samples were prepared in each subject from the same anatomical regions, including the hippocampus and parietal lobe cortex. Samples were embedded into paraffin blocks after fixing. Five-micron-thick sections were taken from paraffin blocks and stained with hematoxylineosin. Sections of each group were evaluated using a camera-attached microscope (Cx43, Olympus, Japan) and images were recorded for further analysis. Samples were evaluated in terms of cellular shrinkage, interstitial edema, vacuolization, and categorical scoring was performed between 0 and 3 (0: None; 1: Mild; 2: Moderate; 3: Severe).

Apoptosis evaluation with TUNEL method: In order to show apoptotic cells in groups, stains were taken after 4-micron-thick sections from paraffin blocks. "ApopTag® Peroxidase In Situ Apoptosis Detection Kit" (Millipore, Merck,
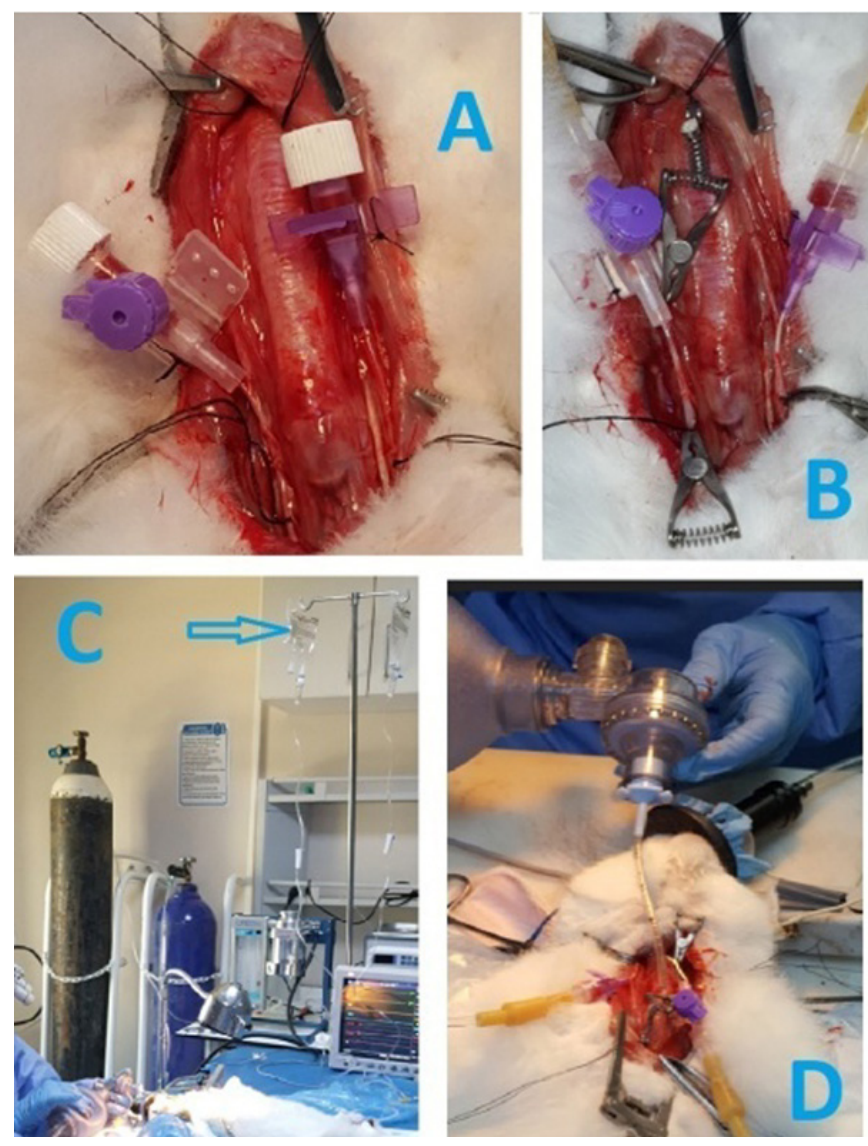

Figure 5. A, the catheters $(26 \mathrm{G})$ placed bilaterally into the common carotid arteries, B, clamps placed over the arteries, C, HTK infusion, $D$, ongoing ischemia.

German) was used. Then, a total of 500 cells were counted under a microscope in five randomly-selected areas of sections taken from each sample. Cells, whose nuclei were stained blue, were considered normal. Those stained brown were considered apoptotic. Apoptotic index was calculated as a percentage (AI).

Statistical analysis: The data of the study were analyzed with the statistical package program SPSS, IBM, Chicago, Illinois, USA 20.0 version. Cell counts, the apoptotic cell index, and histopathological scores were represented as percentages, mean and standard deviation, and median, minimum and maximum values, respectively. ANOVA test with Tukey Post-Hoc analysis was used in normally distributed data. Chi-square test was used in categorical data. Statistical significance was accepted when $P<.05$.

\section{RESULTS}

The apoptotic cell index obtained by the TUNEL method is shown in Table 1 . The number of cells developing apoptosis was high in the ischemia group $(P<.001)$. Post-Hoc analysis showed that the sham and HTK groups were significantly different from the ischemia group, while the HTK and sham groups were similar (Table 1). 
Table 1. Statistical results of TUNEL test

\begin{tabular}{|c|c|c|c|c|}
\hline Sham & $17.8 \pm 6.1$ & - & .000 & .697 \\
\hline HTK & $20.6 \pm 6.2$ & .697 & .000 & - \\
\hline
\end{tabular}

P: ANOVA test (Post-Hoc: Tukey)

Histopathological images obtained by the camera attached to the microscope show apoptotic cells with a blue arrow (Figure 6). Similar to the sham group, there are significantly fewer apoptotic cells in the HTK group than in the ischemia group.

Median scores of qualitative ischemia assessment were 0.5 , 2.0 and 1.0 for cortical vacuolization $(P<.001)$; $0.0,3.0$ and 1.0 for cortical interstitial edema $(P<.001) ; 0.0,1.5$ and 2.0 for cortical cellular shrinkage $(P=.003) ; 0.5,2.5$ and 1.5 for hippocampal vacuolization $(P=.001) ; 0.0,2.5$ and 1.0 for hippocampal interstitial edema $(P<.001)$; and $0.0,2.0$ and 1.0 for hippocampal cellular shrinkage $(P=.001)$ in the sham, ischemia and HTK groups, respectively.

The degree of histopathologic changes was detected as none to mild in all subjects in the sham group, while it was mostly severe in the ischemia group and mild to moderate in the HTK group. Distribution of the scores are represented in Table 2. There was a statistically significant difference between all groups $(P<.05)$.

Interstitial edema, cellular shrinkage, and vacuolization were more intense in the ischemia group (Figure 7).

\section{DISCUSSION}

Ischemic brain damage is a major health condition that can be life-threatening or severely disabling. Protection of the brain against ischemic tissue damage by various methods in the surgeries compromising cerebral circulation for a period is a vital issue. There are different methods suggested for this purpose, especially in carotid interventions. These include hypothermic circulatory arrest [Gokalp 2019], antegrade [Iscan 2018] and retrograde selective cerebral perfusion [Okita 2015] methods, and use of shunts, which still is a topic discussed in carotid surgery [Goodney 2012]. However, despite all these methods, brain damage still may develop. There are current multicenter studies reporting stroke rates around $3.4 \%$ following carotid endarterectomy (CEA) performed, according to current recommendations in patients with high anatomical risk, and stating that these stroke rates are above the 3\%-threshold level and are unacceptable [Rao 2020]. In another study about arcus surgery, which is another condition where cerebral circulation is at risk, it is reported that asymptomatic brain lesions are detected on diffusion MR investigation in $45 \%$ to $100 \%$ of the patients who had surgery with a brain-protection technique [Leshnower 2019]. These findings indicate that although many techniques are available for the protection of the brain, there is still a risk. At this point, in addition to the protection technique methods, selectively increasing the resistance of the brain to ischemia may provide a beneficial option for the cases with both global and local ischemia.

Apoptosis is an important pathological event in cerebral ischemia/reperfusion injury [Bai 2016]. Excessive mitochondrial fission increases mitochondrial external membrane permeability and cytochrome c release, therefore activating the apoptotic cascade and aggravating neurological damage [MacDougall 2018]. HTK solutions establish the resistance of cells to ischemia via buffering of acidosis during anaerobic metabolism by histidine, enhancing ATP production during reperfusion by ketoglutarate, stabilizing the cell membrane by tryptophan, and reducing cellular edema and scavenging free radicals by mannitol [Bretschneider 1980]. In previous studies, it was shown that resistance to ischemia in the heart, kidney, liver and pancreatic cells increased by means of these effects [Agarwal 2006; Edelman 2013; Englesbe 2006; Prathanee 2015; Ringe 2005; Voigt 2013]. Through the same effects, a protective effect can be established against the ischemia-reperfusion damage in the brain cells.

Development of apoptosis and neuron damage may be reduced. By the same physiologic pathway, in the cases with high risk, infusion of tissue-preserving solutions throughout the cerebral circulation may be beneficial to increase the resistance of brain tissue to ischemia.

If the brain-preserving solution is efficient enough, it may be beneficial in high-risk surgeries (CAE, arcus surgery, DHSA, etc.). In addition, it may have some beneficial effects in non-surgical patients with acute stroke similar to cerebral hypothermia obtained by cold saline infusion during angiography, which is proposed by Chen et al [Chen 2016]. In cases of acute brain damage, the application of selective hypothermia was shown to be effective [Kurisu 2016; Yenari 2012]. But, the possible risks of the method should be considered [Wei 2016]. Another known effect of tissue-preserving solutions is that they are protective against hypothermic damage as well [Lautenschläger 2018]. For this reason, tissue-preserving solutions may be a good combination in hypothermic brain protection. It may serve as an important option for the treatment of stroke [Karikari 2018], which is the second most common cause of death following ischemic heart disease.

The aim of this study was to assess whether HTK solution infused throughout the cerebral circulation reduces the degree of brain damage induced by experimental ischemia 
Table 2. Histopathologic scores representing ischemic changes in each group according to the sampling site

\begin{tabular}{|c|c|c|c|c|}
\hline Categoric Variables & Median $(\min / \max )$ & Median $(\min / \max )$ & Median $(\min / \max )$ & $P$-value \\
\hline \multicolumn{5}{|l|}{ Cortical } \\
\hline Vacuolization & $0.5(0-1)$ & $2.0(2-3)$ & $1.0(1-3)$ & 0.000 \\
\hline Cellular shrinkage & $0.0(0-1)$ & $1.5(1-3)$ & $2.0(0-2)$ & 0.003 \\
\hline \multicolumn{5}{|l|}{ Hippocampus } \\
\hline Vacuolization & $0.5(0-1)$ & $2.5(2-3)$ & $1.5(0-3)$ & 0.001 \\
\hline Interstitial edema & $0.0(0-1)$ & $2.5(2-3)$ & $1.0(1-3)$ & 0.000 \\
\hline Cellular shrinkage & $0.0(0-1)$ & $2.0(1-3)$ & $1.0(1-2)$ & 0.001 \\
\hline
\end{tabular}

in an animal model. Both the carotid and subclavian arteries were occluded bilaterally for 15 minutes to produce global brain ischemia followed by a 30-minute reperfusion period [Shintani 2017]. Results of histopathological analysis of the ischemia group revealed that 15-minute occlusion of the bilateral carotid and subclavian arteries is enough to create comparable brain damage in the rabbits.

HTK solution is used as a tissue-protective agent against ischemic damage in heart, kidney and pancreas transplantations, and as a cardioplegic solution in open heart surgery. Its dosage, temperature, application duration, and pressure vary between procedures. The median weight of rabbit brains in the ischemia and sham groups was 8 grams (range 6-10 gr). HTK solution was infused at a rate of $1 \mathrm{~mL} / \mathrm{g} / \mathrm{min}$ for $8-10$ minutes from a height of $50-70 \mathrm{~cm}$ to produce $40-50 \mathrm{mmHg}$ infusion pressure [Minami 2003]. The most difficult part of the experiment was to send HTK solution appropriately into the cerebral circulation. Cannulas were placed within the common carotid artery, and the external carotid arteries were clamped. In this way, the HTK solution was provided to run through only the internal carotid arteries.

Different areas of the brain are variously affected from ischemia. For that reason, histopathological studies were performed on samples taken from both the hippocampus, which is one of the most affected places [Lazarewicz 1989], and the cortex. The severity of ischemia was evaluated using a total of seven histopathological criteria, including cortical cellular shrinkage, cortical interstitial edema, cortical vacuolization, hippocampal cellular shrinkage, hippocampal interstitial edema, hippocampal vacuolization, and apoptotic cell index by TUNEL method.

The significant difference seen in the apoptotic cell indices between the sham and ischemia groups confirmed the occurrence of expected ischemia and the validity of the experimental protocol. According to the apoptotic cell index results, the HTK group was similar to the sham group $(P=.697)$, but not the ischemia group $(P<.001)$. The protective effect of HTK solutions against experimental cerebral ischemia may be concluded, due to similar histopathologic images in the rabbits with and without ischemic insult.
The median values of histopathological scores showing the degree of ischemic brain damage were significantly different between groups and were highest in the ischemia group. They were lower in the HTK group than the ischemia group, representing lower ischemic damage with HTK solution, despite similar vascular occlusion protocol.

Samples taken from both the cortical and hippocampal regions were examined using four separate histopathological ischemic findings (AI, interstitial edema, cellular shrinkage, and vacuolization). This approach provided a wider examination area to detect the presence and degree of ischemia. When both TUNEL assay and the scoring results were examined, it was observed that ischemic findings occurred in all groups (Tables 1 and 2). Also the sham group, which did not experience the vascular occlusion, had ischemic findings (AI:17.8, Table 2). This finding was attributed to the sensitivity of nerve cells to ischemia and the process during the brain extraction phase. As expected, the most severe ischemic findings occurred in the ischemia group (AI:55.8, Table 2). In the study conducted by Iwama et al, it was shown that ischemic findings did not occur in the brains of rabbits although the bilateral carotid arteries of their rabbits were clamped, and that $50 \%$ necrosis occurred after the left subclavian artery was clamped [Iwama 2000]. This may be attributed to the strong collateral circulation of the rabbit brain. The degree of ischemia was severe in the rabbits with occlusion of the bilateral carotid and subclavian arteries (AI:55.8, Table 2). The data obtained from the HTK group revealed that AI was 20.6, and scores were mild to moderate degree. These results were close to that of the sham group, where ischemia was not induced, and there was not statistical difference $(P=.697)$. There was a high level of statistical difference compared with the ischemia group $(P<$ .0001). These results indicate that the direct addition of HTK solution to cerebral circulation decreases the degree of ischemic damage. In the present study, the solution applied was hypothermic $\left(4-10^{\circ} \mathrm{C}\right)$. One of the most important issues to be clarified is the contribution of hypothermia on cerebral protection in this setting. Among the limited number of studies in the literature, Kang et al revealed that the cold $\left(4^{\circ} \mathrm{C}\right) \mathrm{HTK}$ 


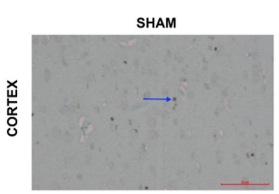

SHAM
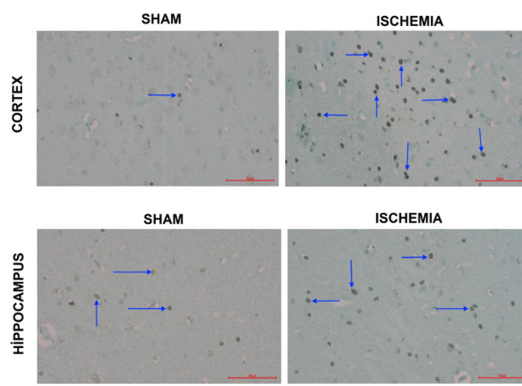

ISCHEMIA
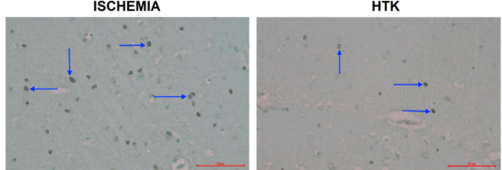

Figure 6. The TUNEL staining of the samples from all experimental groups. Blue arrows are marking the apoptotic cells ( $\times 400$ magnification). Samples were stained with hematoxylin-eosin.

solution had a superior neuroprotective effect compared with cold $\left(4^{\circ} \mathrm{C}\right)$ saline in rats with spinal cord ischemia [Kang 2016]. In the study conducted by Hsu et al, neuroprotection with the normothermic HTK solution against ischemia-reperfusion damage in rats with asphyxia was revealed [Hsu 2018]. Hoyer et al revealed that less brain damage occurred with the use of the HTK-N cardioplegia in an arrest model of pigs [Hoyer 2019]. Because it has protective effect against cold damage [Lautenschläger 2018], the HTK solution may serve as a suitable agent for hypothermic applications. It has been suggested by previous studies that hypothermia may enhance the protective effect of the HTK solutions [Choi 2018; Englum 2013]. Additionally, all tissue-preserving solutions are expected to be more efficient with the suitable combination of hypothermia. Similarly, the effects of tissue-preserving solutions may be utilized to create safe temperature ranges in hypothermia applications. Future studies investigating the effects of various temperatures, dosages and durations are needed to clarify their exact effects.

It was determined that the brain was less affected by ischemia in the HTK group. Translation of these positive results into clinical setting is far away for the moment and requires further investigations. But it is a new idea, and the current experimental study revealed promising results.

Study limitations: Brain temperature was not measured in this study, which can be changed during administration of HTK solution and may have an important role in cerebral protection and brain damage. Another limitation is that the brain damage was assessed by only visual histopathologic evaluations, providing mostly rough categorical data. More sophisticated methods should be used to determine the degree of brain damage as well as brain functions.

\section{CONCLUSION}

In conclusion, it is observed that application of HTK solution directly into cerebral circulation during carotid occlusion significantly reduces brain damage caused by experimental ischemia. Further studies are needed to show the effect of HTK solution on brain temperature and protection capability and to assess brain functions and damage with more sophisticated methods other than visual histopathologic evaluation alone.

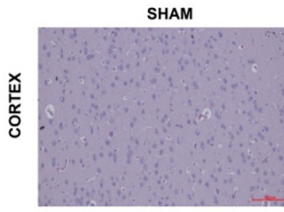

SHAM
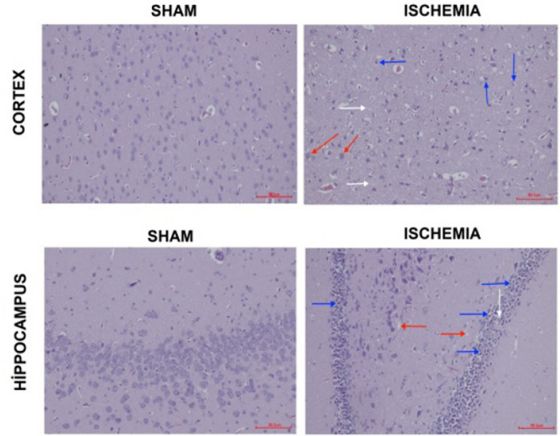

ISCHEMIA
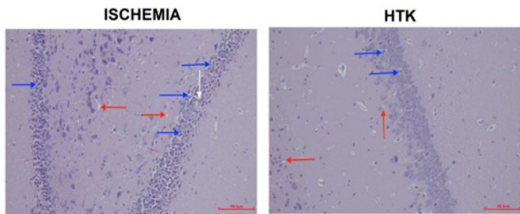

Figure 7. The figure reveals histopathologic changes in the groups. Interstitial edema (red arrows), cellular shrinkage (blue arrows), and vacuolization (white arrows) areas are marked in each cortical section ( $\times 200$ magnification). Samples were stained with hematoxylin-eosin.

\section{ACKNOWLEDGEMENT}

This study was supported by Canakkale Onsekiz Mart University Scientific Research Project Coordination Center. Project number: TSA-2019-2929. We would like to thank veterinarian doctor Sait Elmas and the entire staff of Canakkale Onsekiz Mart University Experimental Research Application and Research Center for their support during surgical procedures. We would like to thank Prof. Dr. Coskun Bakar and Dr. Özgür Özerdoğan for support in statistical analyses.

\section{REFERENCES}

Agarwal A, Murdock P, Fridell JA. 2006. Comparison of histidinetryptophan ketoglutarate solution and University of Wisconsin solution in prolonged cold preservation of kidney allografts. Transplantation 81:480-482.

Arslan A, Sezgin A, Gultekin B, Ozkan S, Akay T, Uguz E, et al. 2005. Low-dose histidine-tryptophan-ketoglutarate solution for myocardial protection. In Transplantation proceedings 37:3219-3222.

Bai S, Sun Y, Wu L, Wu Z, Fang M. 2016. Tripotolide ameliorates inflammation and apoptosis induced by focal cerebral ischemia/reperfusion in rats. Med JZU 45:493-500.

Bretschneider HJ. 1980. Myocardial protection. Thorac Cardiovasc Surg 28:295-302.

Chen J, Liu L, Zhang H, Geng X, Jiao L, Li G, et al. 2016. Endovascular hypothermia in acute ischemic stroke: pilot study of selective intraarterial cold saline infusion. Stroke 47:1933-1935.

Choi JH, Pile-Spellman J. 2018. Selective brain hypothermia. In Handbook of clinical neurology 157:839-852.

Edelman JJ, Seco M, Dunne B, Matzelle SJ, Murphy M, Joshi P, et al. 2013. Custodiol for myocardial protection and preservation: a systematic review. Ann Cardiothorac Surg 2:717-28.

Englesbe MJ, Moyer A, Kim DY, Granger DK, Pietroski R, Yoshida A, et al. 2006. Early Pancreas Transplant Outcomes with Histidine-Tryptophan-Ketoglutarate Preservation: A Multicenter Study, Transplantation 82:136-13. 
Englum BR, Andersen ND, Husain AM, Mathew JP, Hughes GC. 2013. Degree of hypothermia in aortic arch surgery-optimal temperature for cerebral and spinal protection: deep hypothermia remains the gold standard in the absence of randomized data. Annals of Cardiothoracic Surgery $2: 184$.

Gokalp O, İner H, Karakas NY, Iscan S, Gokalp G, Besir Y, et al. 2019. Deep Hypothermic Circulatory Arrest in Aortic Dissection Surgery. Heart, Lungand Circulation 28:10-11.

Goodney PP, Wallaert JB, Scali ST, Stone DH, Patel V, Shaw P, et al. 2012. Impact of practice patterns in shunt use during carotid endarterectomy with contralateral carotid occlusion. Journal of Vascular Surgery $55: 61-71$.

Hoyer A, Then BF, Klaeske K, Lehmann S, Misfeld M, Borger M, et al. 2019. Custodiol- $\mathrm{N}^{\mathrm{TM}}$ cardioplegia lowers cerebral inflammation and activation of hypoxia-inducible factor- $1 \alpha$. Interactive Cardiovascular and Thoracic Surgery 28:884-892.

Hsu J, Wang C, Huang S, Chen Y, Yu S, Huang J, et al. 2018. HistidineTryptophan-Ketoglutarate Solution as a Neuroprotective Against Ischemia/Reperfusion Injury. Preprints, 2018100105.

Iscan S, Eygi B. 2018. Neurologic and Renal Outcomes of Elective Proximal Aortic Repair and Current Cannulation Trends. Int J Clin Cardiol $5: 128$.

Iwama H, Akama Y, Tase C. 2000. Global brain ischemia produced by clamping left subclavian artery and bicarotid trunk in the rabbit. The American Journal of Emergency Medicine 18:31-35.

Kalogeris T, Baines CP, Krenz M, Korthuis RJ. 2012. Cell biology of ischemia/reperfusion injury. In International review of cell and molecular biology. Academic Press 298:229-317.

Kang SK, Kang MW, Rhee YJ, Kim CS, Jeon BH, Han SJ, et al. 2016. In vivo neuroprotective effect of histidine-tryptophan-ketoglutarate solution in an ischemia/reperfusion spinal cord injury animal model. The Korean Journal of Thoracic and Cardiovascular Surgery 49:232.

Karikari TK, Charway-Felli A, Höglund K, Blennow K, Zetterberg H. 2018. Commentary: global, regional, and national burden of neurological disorders during 1990-2015: a systematic analysis for the Global Burden of Disease Study 2015. Frontiers in Neurology 9:201.

Kurisu K, Abumiya T, Ito M, Gekka M, Osanai T, Shichinohe H, et al. 2016. Transarterial regional hypothermia provides robust neuroprotection in a rat model of permanent middle cerebral artery occlusion with transient collateral hypoperfusion. Brain Res 1651:95-103.

Lautenschläger I, Pless-Petig G, Middel P, de Groot H, Rauen U, Stojanovic T. 2018. Cold Storage Injury to Rat Small-bowel Transplants-Beneficial Effect of a Modified HTK Solution. Transplantation 102:1666-1673.

Lazarewicz JW, Pluta R, Salinska E, Puka M. 1989. Beneficial effect of nimodipine on metabolic and functional disturbances in rabbit hippocampus following complete cerebral ischemia. Stroke 20:70-77.

Lee S, Huang CS, Kawamura T, Shigemura N, Billiar TR, Nakao A, et al. 2011. Histidine-tryptophan-ketoglutarateor Celsior: which is more suitable for cold preservation for cardiac grafts from older donors.
AnnThorac Surg 91:755-763.

Lee S, Huang CS, Kawamura T, Shigemura N, Stolz DB, Billiar TR, et al. 2010. Superior myocardial preservation with HTK solution over Celsior in rat hearts with prolonged cold ischemia. Surgery 148:463-473.

Leshnower BG, Rangaraju S, Allen JW, Stringer AY, Gleason TG, Chen EP. 2019. Deep hypothermia with retrograde cerebral perfusion versus moderate hypothermia with antegrade cerebral perfusion for arch surgery. The Annals of Thoracic Surgery 107:1104-1110.

MacDougall G, Anderton RS, Mastaglia FL, Knuckey NW, Meloni BP. 2018. Mitochondria and neuroprotection in stroke: cationic argininerich peptides (CARPs) as a novel class of mitochondria-targeted neuroprotective therapeutics. Neurobiol Dis 121:17-33.

Michel P, Vial R, Rodriguez C, Ferrera R. 2002. A comparative study of the most widely used solutions for cardiac graft preservation during hypothermia. The Journal of heart and lung transplantation 21:1030-1039.

Minami K, Omoto T, Böthig D, Tenderich G, Wlost S, Schütt U, et al. 2003. Creatine kinase and troponin after myocardial preservation using HTK solution (Custoidol) for clinical heart transplantation. The Journal of Heart and Lung Transplantation 22:192-194.

Okita Y, Miyata H, Motomura N, Takamoto S, \& Organization T. J. C. S. D. A 2015. Study of brain protection during total arch replacement comparing antegrade cerebral perfusion versus hypothermic circulatory arrest, with or without retrograde cerebral perfusion: analysis based on the Japan Adult Cardiovascular Surgery Database. The Journal of Thoracic and Cardiovascular Surgery 149:65-73.

Prathanee S, Kuptanond C, Intanoo W, Wongbhudha C, Karunasumaeta C. 2015. Custodial-HTK Solution for Myocardial Protection in CABG Patients. J MedAssocThai 98:164-7.

Rao V, Liang P, Swerdlow N, Li C, Solomon Y, Wyers M, et al. 2020. Contemporary outcomes after carotid endarterectomy in high-risk anatomic and physiologic patients. Journal of Vascular Surgery 71:104-110.

Ringe B, Braun F, Moritz M, Zeldin G, Soriano H, Meyers W. 2005. Safety and efficacy of living donor liver preservation with HTK solution. In Transplantation proceedings 37:316-319.

Saitoh Y, Hashimoto M, Ku K, Kin S, Nosaka S, Masumura S, et al. 2000. Heartpreservation in HTK solution: role of coronary vasculature in recovery of cardiacfunction. Ann Thorac Surg 69:107-112.

Shintani N, Ishiyama T, Kotoda M, Asano N, Sessler DI, Matsukawa T. 2017. The effects of Y-27632 on pial microvessels during global brain ischemia and reperfusion in rabbits. BMC Anesthesiology 17:38.

Voigt MR, Ginger TD. 2013. Perspectives on abdominal organ preservation solutions: a comparative literature review. Progress in Transplantation 23:383-391.

Wei X, Hu CC, Zhang YL, Yao SL, Mao WK. 2016. Telmisartan reduced cerebral edema by inhibiting NLRP 3 inflammasome in mice with cold brain injury. Journal of Huazhong University of Science and Technology. Medical Sciences 36:576-583.

Yenari MA, Han HS. 2012. Neuroprotective mechanisms of hypothermia in brain ischaemia. Nat Rev Neurosci 13:267-278. 